\title{
Ground State Energies of Helium-Like Ions Using a Simple Parameter-Free Matrix Method
}

\author{
Redi Kristian Pingak ${ }^{*}$, Atika Ahab ${ }^{1}$, and Utama Alan Deta ${ }^{2}$ \\ ${ }^{1}$ Department of Physics, Universitas Nusa Cendana, Jl. Adisucipto Penfui, Kupang 85001, Nusa Tenggara Timur, Indonesia \\ ${ }^{2}$ Department of Physics, Universitas Negeri Surabaya, Jl. Ketintang Gd C3 Lt 1, Surabaya 60231, Indonesia
}

\section{* Corresponding author:}

tel: $+62-82147618836$

email: rpingak@staf.undana.ac.id

Received: May 6, 2021

Accepted: June 7, 2021

DOI: $10.22146 / \mathrm{ijc} .65737$

\begin{abstract}
This study aims to use hydrogenic orbitals within an analytic and numeric parameter-free truncated-matrix method to solve the projected Schrödinger equation of some helium-like ions $(3 \leq Z \leq 10)$. We also derived a new analytical expression of the ion ground state energies, which was simple and accurate and improved the accuracy of the analytic calculation, numerically using Mathematica. The standard matrix method was applied, where the wave function of the ions was expanded in a finite number of eigenvectors comprising hydrogenic orbitals. The Hamiltonian of the systems was calculated using the wave function and diagonalized to obtain their ground state energies. The results showed that a simple analytic expression of the ground state energies of Helike ions was successfully derived. Although the analytic expression was derived without involving any variational parameter, it was reasonably accurate with a $0.12 \%$ error for $\mathrm{Ne}^{8+}$ ion., The accuracy of the analytic energies was also numerically improved to $0.10 \%$ error for $\mathrm{Ne}^{8+}$ ion from this method. The results clearly showed that the energies obtained using this method were more accurate than the hydrogenic perturbation theory and the uncertainty principle-variational approach. In addition, for $Z>4$, our results were more accurate than those from the geometrical model.
\end{abstract}

Keywords: helium-like ions; ground state energies; parameter-free matrix method; hydrogenic orbital approximation; projected Schrödinger equation

\section{- INTRODUCTION}

Theoretical calculations of energies of two-electron systems such as helium and helium-like ions have been attractive since the discovery of quantum mechanics [1] because these systems are the most simple many-body systems, and therefore, traditionally used as a testing ground for various methods in theoretical quantum calculations [2]. In addition, highly accurate experimental data for such systems are available, which can be used as a reference to evaluate the accuracy of theoretical calculations.

A large number of theoretical studies have been conducted to calculate the energies of helium-like ions accurately. The methods used in the studies ranged from the most sophisticated to the simplest ones. Some relatively new advanced calculations were quantum electrodynamics calculations [2-3], explicitly correlated basis sets derived from regularized Krylov sequences [46] and discrete variational-perturbation approach based on explicitly correlated wave functions [7-8]. Furthermore, some highly accurate calculations on the energies of the ions using various methods were recently reported, including the application of the free iterative complement interaction method [9-10], NonRelativistic QED (NRQED) [11], and Quantum Mechanics of Coulumb Charge (QMCC) [12]. Older sophisticated calculations include the work of Pekeris, who applied the iteration method with the use of perimetric coordinates in the wave function expansion [13]. In general, up to thousands of variational parameters were used in most sophisticated calculations to produce highly accurate energies of helium-like ions [14]. However, these methods mostly involved tedious analytical calculations and or expensive numerical 
calculations, mainly due to many parameters in their calculations. Moreover, wavefunctions obtained from such methods are often computationally prohibitive to be used in calculating cross-sections of many physical processes such as double ionization of atoms by ion [14]. Therefore, finding simpler wavefunctions with a low number of parameters is essential for studying those physical processes.

In addition to the methods mentioned above, several relatively more straightforward methods have also been developed recently by reducing the number of variational parameters. For instance, Liverts and Barnea [15] performed numerical calculations, which only used three parameters as inputs in their Mathematica code. Some analytical calculations using trial wave functions with a minimum number of parameters were also developed [16]. The final form of the proposed wavefunction can be written as a parameter-free function. However, the calculations to obtain its final form still involved optimizing a parameter in the initially proposed wavefunction. A numerical calculation using the variational method was also applied in [17]. Moreover, a parameter-free-like method using a simple geometrical model was developed by Gomez [18] to calculate the energies of helium-like ions. In this geometrical model, there was only a single parameter used in the calculations, but errors were still relatively large, especially for ions with large Z.

The simplest possible method to calculate the energies of helium-like ions is to use the hydrogenic orbital approximation. In this method, two-electron wave functions of the ions are written as the Hartree product of two hydrogenic orbitals. In this approach, correlated wave functions, which often make theoretical calculations more complicated, are neglected, and no variational parameter is needed [19]. However, the ground state energy results have relatively large errors, especially for the ions with relatively small atomic number $Z$, which indicates a large discrepancy between results obtained from the simplest methods with those from the most accurate ones. Hence, finding a simple yet accurate method to calculate energies of two-electron atoms such as helium-like ions remains a significant challenge.
To increase the accuracy of the hydrogenic standard perturbation theory, a simple numerical calculation using the hydrogenic orbital approximation in the framework of a matrix calculation was introduced by Masse and Walker [20]. In their work, a numerical calculation using a Mathematica code containing only seven lines was applied to calculate the three lowest energies of the helium atom with reasonable accuracy. Although the method did not require any parameter in the calculation, it managed to significantly reduce errors obtained from the standard $1^{\text {st }}$ order perturbation theory. Furthermore, a simple analytical matrix method, based on the work of [20], was developed in our previous work [21] to obtain accurate analytical energies of the helium atom. The numerical and analytical matrix approach applied in the two studies performed well for helium and yielded much more accurate energies than those from the $1^{\text {st }}$ order standard hydrogenic perturbation theory. This method was also applied recently in our previous study to obtain the first excited state energies of some helium-like ions with reasonable accuracy [22].

To the best of our knowledge, the parameter-free matrix method has not been used to calculate the ground state energies of helium-like ions. Also, since the method has proven accuracy when used with the helium atom [20-21], it should perform better if applied to other twoelectron systems such as helium-like ions with larger atomic numbers than the helium atom.

The purpose of the present study was to extend the parameter-free matrix method to obtain accurate ground state energies of light helium-like ions $(3 \leq \mathrm{Z} \leq$ 10) with analytic and numeric calculations. The Mathematica code of Masse and Walker [20] and the analytic matrix method [21] were used for the numerical and analytical calculations, respectively. In this work, only s-orbitals were included in the wave functions to avoid long calculations. To demonstrate the ease of the method, a simple analytic expression for energies of any helium-like ion, which only depends on the atomic number Z, was derived in this work. This analytical expression can then be used as an easy-to-use equation to calculate the energies of helium-like ions. With this 
parameter-free and straightforward method, this work aims not to better highly advanced variational calculation methods but to produce more accurate ground state energies of helium-like ions than other simple approaches in the literature, with much less algebra and minimum computational time. Hartree atomic units were used throughout this work.

\section{- COMPUTATIONAL METHODS}

The parameter-free matrix method solved the projected Schrödinger equation in a finite subspace. The Hamiltonian was in the form of a finite square matrix, and the wave functions were in the form of a column vector. The Hamiltonian matrix elements were calculated after the wave function was defined to solve the energy eigenvalue equation. In this work, the two-electron wave function of the ions was written as a linear combination of basis states formed from the Hartree product of two hydrogenic orbitals. Once the Hamiltonian matrix elements were found, the last step was to solve the secular equation to obtain energy eigenvalues of the ions. The ground state energy was the smallest among the energy eigenvalues obtained. The formulations needed to perform those calculations are presented below. The nonrelativistic time-independent Schrödinger equation projected into a finite subspace for two-electron atoms (ions) with a fixed nucleus is

$\hat{\mathrm{H}}|\Psi\rangle=\mathrm{E}|\Psi\rangle$

or explicitly as

$\left(-\frac{\nabla_{1}^{2}}{2}-\frac{\nabla_{2}^{2}}{2}-\frac{\mathrm{Z}}{\mathrm{r}_{1}}-\frac{\mathrm{Z}}{\mathrm{r}_{2}}+\frac{1}{\mathrm{r}_{12}}\right)|\Psi\rangle=\mathrm{E}|\Psi\rangle$

where $r_{1}$ and $r_{2}$ are the nuclear-electron distance of electron 1 and electron 2, respectively, $r_{12}$ is the electronelectron distance, and $\mathrm{Z}$ is the atomic number of the ions. In the orbital approximation, the wavefunction in Eq. (2) can be written as a linear combination of the Hartree product of hydrogenic orbitals as

$|\Psi\rangle=\sum_{\mathrm{i}=1}^{\mathrm{n}} \mathrm{c}_{\mathrm{i}}\left|\Phi_{\mathrm{i}}\right\rangle=\sum_{\mathrm{i}=2}^{\mathrm{n}} \mathrm{c}_{11}\left|\phi_{1} \phi_{1}\right\rangle+\mathrm{c}_{1 \mathrm{i}}\left|\phi_{1} \phi_{\mathrm{i}}\right\rangle+\mathrm{c}_{\mathrm{i} 1}\left|\phi_{\mathrm{i}} \phi_{1}\right\rangle$

where $\varphi_{1}$ is the hydrogenic orbital of an electron in the ground state and $\varphi_{\mathrm{i}}$ is that of the other electron, which can be in any state of the helium-like ions. The number of terms in the expansion depends on the number of basis states one wants to use in the calculation. Elements of the Hamiltonian matrix is determined using Eq. (4)

$\mathrm{H}_{\mathrm{pq}}=\left\langle\Phi_{\mathrm{p}}|\mathrm{H}| \Phi_{\mathrm{q}}\right\rangle$

substituting the explicit form of Hamiltonian in Eq. (2) into Eq. (4) yielded

$\mathrm{H}_{\mathrm{pq}}=\left(-\frac{\mathrm{Z}^{2}}{2 \mathrm{n}_{1}^{2}}-\frac{\mathrm{Z}^{2}}{2 \mathrm{n}_{2}^{2}}\right) \delta_{\mathrm{pq}}+\left\langle\Phi_{\mathrm{p}}\left|\frac{1}{\mathrm{r}_{12}}\right| \Phi_{\mathrm{q}}\right\rangle$

where the first two terms in Eq. (5) are the two hydrogenic one-electron solutions of the non-relativistic Schrödinger equation. The electron-electron interaction potential in Eq. (5) can be determined using Eq. (6)

$\mathrm{V}=\frac{1}{\mathrm{r}_{12}}=\sum_{\mathrm{lm}} \frac{4 \pi}{2 \mathrm{l}+1} \frac{\mathrm{r}_{<}{ }^{1}}{\mathrm{r}_{>}^{1+1}}(-1)^{\mathrm{m}} \mathrm{Y}_{1}^{-\mathrm{m}}\left(\Omega_{1}\right) \mathrm{Y}_{1}^{\mathrm{m}}\left(\Omega_{2}\right)$

$\mathrm{V}=\left\{\begin{array}{l}\frac{1}{\mathrm{r}_{1}}, \text { if } \mathrm{r}_{2}<\mathrm{r}_{1} \\ \frac{1}{\mathrm{r}_{2}}, \text { if } \mathrm{r}_{1}<\mathrm{r}_{2}\end{array}\right.$

Each hydrogenic orbital $\left|\varphi_{\mathrm{i}}\right\rangle$ used in the basis states $\left|\Phi_{\mathrm{p}}\right\rangle$ is the product of the radial function and the spherical harmonics. Since only s-orbitals were used in this article, the orbital can then be written as

$\left|\phi_{\mathrm{n} 00}(\mathrm{r}, \theta, \phi)\right\rangle=\left|\mathrm{R}_{\mathrm{n} 0}(\mathrm{r}) \mathrm{Y}_{0}^{0}\right\rangle$

where the well-known hydrogenic radial function for sorbitals can be written in terms of Laguerre polynomials as

$\mathrm{R}_{\mathrm{n} 0}\left(\mathrm{r}_{\mathrm{i}}\right)=\left\{\frac{\mathrm{Z}(\mathrm{n}-1) !}{\mathrm{n}^{2}(\mathrm{n}) !}\right\}^{\frac{1}{2}}\left(\frac{2 \mathrm{Z}}{\mathrm{n}}\right) \mathrm{e}^{-\frac{\mathrm{Z} \mathrm{r}_{\mathrm{i}}}{\mathrm{n}}} \mathrm{L}_{\mathrm{n}-1}^{1}\left(\frac{2 \mathrm{Zr}_{\mathrm{i}}}{\mathrm{n}}\right)$

Substituting Eq. (7) and (8) into Eq. (5) and integrating it over all space yields

$$
\begin{aligned}
\mathrm{H}_{\mathrm{pq}}= & \left(-\frac{\mathrm{Z}^{2}}{2 \mathrm{n}_{1}{ }^{2}}-\frac{\mathrm{Z}^{2}}{2 \mathrm{n}_{2}{ }^{2}}\right) \delta_{\mathrm{pq}}+\left(\mathrm{Y}_{0}^{0}\right)^{4} \iint \mathrm{R}_{\mathrm{n}_{1} 0}\left(\mathrm{r}_{1}\right) \mathrm{R}_{\mathrm{n}_{2} 0}\left(\mathrm{r}_{2}\right) \\
& \frac{1}{\mathrm{r}_{12}} \mathrm{R}_{\mathrm{n}_{3} 0}\left(\mathrm{r}_{1}\right) \mathrm{R}_{\mathrm{n}_{4} 0}\left(\mathrm{r}_{2}\right) \mathrm{d}^{3} \mathrm{r}_{1} \mathrm{~d}^{3} \mathrm{r}_{2}
\end{aligned}
$$

Since the angular part of the integrations canceled $\left(\mathrm{Y}_{0}^{0}\right)^{4}$, Eq. (9) becomes

$$
\begin{aligned}
\mathrm{H}_{\mathrm{pq}}= & \left(-\frac{\mathrm{Z}^{2}}{2 \mathrm{n}_{1}^{2}}-\frac{\mathrm{Z}^{2}}{2 \mathrm{n}_{2}^{2}}\right) \delta_{\mathrm{pq}}+\iint \mathrm{r}_{1}^{2} \mathrm{R}_{\mathrm{n}_{1} 0}\left(\mathrm{r}_{1}\right) \mathrm{R}_{\mathrm{n}_{2} 0}\left(\mathrm{r}_{2}\right) \\
& \frac{1}{\mathrm{r}_{12}} \mathrm{r}_{2}^{2} \mathrm{R}_{\mathrm{n}_{3} 0}\left(\mathrm{r}_{1}\right) \mathrm{R}_{\mathrm{n}_{4} 0}\left(\mathrm{r}_{2}\right) \mathrm{dr}_{1} \mathrm{dr}_{2}
\end{aligned}
$$

By substituting Eq. (8) into Eq. (10), $\mathrm{r}_{1}^{2}$ and $\mathrm{r}_{2}^{2}$ can be 
absorbed into the radial functions, and therefore, Eq. (10) has a simpler form as

$$
\begin{aligned}
\mathrm{H}_{\mathrm{pq}}= & \left(-\frac{\mathrm{Z}^{2}}{2 \mathrm{n}_{1}^{2}}-\frac{\mathrm{Z}^{2}}{2 \mathrm{n}_{2}^{2}}\right) \delta_{\mathrm{pq}}+\iint \mathrm{P}_{\mathrm{n}_{1} 0}\left(\mathrm{r}_{1}\right) \mathrm{P}_{\mathrm{n}_{2} 0}\left(\mathrm{r}_{2}\right) \\
& \frac{1}{\mathrm{r}_{12}} \mathrm{P}_{\mathrm{n}_{3} 0}\left(\mathrm{r}_{1}\right) \mathrm{P}_{\mathrm{n}_{4} 0}\left(\mathrm{r}_{2}\right) \mathrm{dr}_{1} \mathrm{dr}_{2}
\end{aligned}
$$

As a consequence, a modified radial function $\mathrm{P}_{n 0}\left(\mathrm{r}_{\mathrm{i}}\right)$ has to be defined as

$\mathrm{P}_{\mathrm{n} 0}\left(\mathrm{r}_{\mathrm{i}}\right) \equiv\left\{\frac{\mathrm{Z}(\mathrm{n}-1) !}{\mathrm{n}^{2}(\mathrm{n}) !}\right\}^{\frac{1}{2}}\left(\frac{2 \mathrm{Zr} \mathrm{r}_{\mathrm{i}}}{\mathrm{n}}\right) \mathrm{e}^{-\frac{\mathrm{Zr} \mathrm{r}_{\mathrm{i}}}{\mathrm{n}}} \mathrm{L}_{\mathrm{n}-1}^{1}\left(\frac{2 \mathrm{Zr} \mathrm{r}_{\mathrm{i}}}{\mathrm{n}}\right)$

where $\mathrm{i}=1,2$. Eq. (11) was the expression used to analytically and numerically calculate elements of the Hamiltonian matrix in this article.

After the Hamiltonian matrix was obtained, the secular equation, see Eq. (13), was simply solved to obtain all energy eigenvalues (assuming the Hamiltonian matrix is of size $j \times j$ ).

$$
\operatorname{det}\left[\begin{array}{cccc}
\mathrm{H}_{11}-\mathrm{E} & \mathrm{H}_{12} & \ldots & \mathrm{H}_{1 \mathrm{j}} \\
\mathrm{H}_{21} & \mathrm{H}_{22}-\mathrm{E} & \ldots & \mathrm{H}_{2 \mathrm{j}} \\
\ldots & \ldots & \ldots & \ldots \\
\mathrm{H}_{\mathrm{j} 1} & \mathrm{H}_{\mathrm{j} 2} & \ldots & \mathrm{H}_{\mathrm{jj}}-\mathrm{E}
\end{array}\right]=0
$$

Solutions to Eq. (13) contain j energy eigenvalues, and the lowest energy corresponds the ground state energy of the He-like ions. In this work, Eq. (11) and (13) were solved both analytically and numerically.

\section{- RESULTS AND DISCUSSION}

\section{Analytic Expression for the Ground State Energies of He-Like lons and Analytical Results}

In this section, an analytic expression for the He-like ion energies was derived using the free-parameter matrix method with three basis states. First, Eq. (3) was used to determine the two-electron wavefunction expanded in three basis states consisting of hydrogenic s-orbitals, as shown in Eq. (14)

$|\Psi\rangle=c_{11}|1 s 1 s\rangle+c_{12}|1 s 2 s\rangle+c_{21}|2 s 1 s\rangle$

Then, the Hamiltonian was in the form of a $3 \times 3$ matrix, whose nine components were determined using Eq. (11). To determine $\mathrm{H}_{11}$, one had

$\mathrm{H}_{11}=-\mathrm{Z}^{2}+\left\langle\mathrm{P}_{10} \mathrm{P}_{10}\left|\frac{1}{\mathrm{r}_{12}}\right| \mathrm{P}_{10} \mathrm{P}_{10}\right\rangle$
$\mathrm{P}_{10}$ was simply determined using Eq. (12), which was then substituted into Eq. (15) to obtain (in integral form)

$$
\mathrm{H}_{11}=-\mathrm{Z}^{2}+2^{4} \mathrm{Z}^{6} \iint \mathrm{r}_{1}^{2} \mathrm{e}^{-2 \mathrm{Zr}_{1}} \frac{1}{\mathrm{r}_{12}} \mathrm{r}_{2}^{2} \mathrm{e}^{-2 \mathrm{Zr}_{2}} \mathrm{dr}_{1} \mathrm{dr}_{2}
$$

To evaluate the integral in Eq. (16), one could rewrite it as

$\mathrm{H}_{11}=-\mathrm{Z}^{2}+2^{4} \mathrm{Z}^{6} \int_{0}^{\infty} \mathrm{r}_{1}^{2} \mathrm{e}^{-2 \mathrm{Zr}_{1}}\left(\mathrm{I}_{2}\right) \mathrm{dr} \mathrm{r}_{1}$

where $\mathrm{I}_{2}$ had been defined to be

$\mathrm{I}_{2} \equiv \int_{0}^{\infty} \frac{1}{\mathrm{r}_{12}} \mathrm{r}_{2}^{2} \mathrm{e}^{-2 \mathrm{Zr}_{2}} \mathrm{dr} \mathrm{r}_{2}$

using Eq. (6) and detail procedures in [21], one obtained

$\mathrm{I}_{2}=\frac{1}{4 \mathrm{Z}^{3} \mathrm{r}_{1}}\left\{\left(-\mathrm{Zr}_{1}-1\right) \mathrm{e}^{-2 \mathrm{Zr}_{1}}+1\right\}$

substituting Eq. (19) back into Eq. (17) yielded

$\mathrm{H}_{11}=-\mathrm{Z}^{2}+4 \mathrm{Z}^{3} \int_{0}^{\infty}\left\{\left(-\mathrm{Zr}_{1}{ }^{2}-\mathrm{r}_{1}\right) \mathrm{e}^{-4 Z \mathrm{Zr}_{1}}+\mathrm{r}_{1} \mathrm{e}^{-2 Z \mathrm{r}_{1}}\right\} d \mathrm{r}_{1}$

Similarly, other elements of the Hamiltonian matrix were obtained using the same procedures involved in obtaining $\mathrm{H}_{11}$. The other elements could be easily proven to be equal to Eq. (21), Eq. (22) and Eq. (23). $\mathrm{H}_{12}=\mathrm{H}_{21}=\mathrm{H}_{13}=\mathrm{H}_{31}$

$$
=\sqrt{2} Z^{3} \int_{0}^{\infty}\left\{\begin{array}{l}
\left(\frac{Z^{2}}{2} r_{1}^{3}-\frac{Z}{2} r_{1}^{2}-r_{1}\right) e^{-7 Z_{1} / 2}+ \\
\left(-\frac{Z}{2} r_{1}^{2}+r_{1}\right) e^{-3 Z r_{1} / 2}
\end{array}\right\} d r_{1}
$$

$$
\mathrm{H}_{23}=\mathrm{H}_{32}=\frac{2 \mathrm{Z}^{4}}{3^{3}} \int_{0}^{\infty}\left(-3 \mathrm{Z}^{2} \mathrm{r}_{1}^{4}+4 \mathrm{Zr}_{1}^{3}+4 \mathrm{r}_{1}^{2}\right) \mathrm{e}^{-3 \mathrm{Zr}_{1}} \mathrm{dr}_{1}
$$

$\mathrm{H}_{22}=\mathrm{H}_{33}$

$$
=\frac{-5 Z^{2}}{8}+\frac{Z^{3}}{2} \int_{0}^{\infty}\left\{\begin{array}{l}
\left(-\frac{Z^{3}}{4} r_{1}^{4}+\frac{3 Z^{2}}{4} r_{1}^{3}-r_{1}\right) e^{-3 Z r_{1}}+ \\
\left(\frac{Z^{2}}{4} r_{1}^{3}-Z r_{1}^{2}+r_{1}\right) e^{-Z r_{1}}
\end{array}\right\} d r_{1}
$$

Eq. (20) to Eq. (23) were then evaluated to obtain all elements of the Hamiltonian matrix, as shown in Eq. (24). All are in Hartree atomic units.

$$
\mathrm{H}=\left[\begin{array}{ccc}
-\mathrm{Z}\left(\mathrm{Z}-\frac{5}{8}\right) & \sqrt{2}\left(\frac{4096}{64827} \mathrm{Z}\right) & \sqrt{2}\left(\frac{4096}{64827} \mathrm{Z}\right) \\
\sqrt{2}\left(\frac{4096}{64827} \mathrm{Z}\right) & -\mathrm{Z}\left(\frac{5}{8} \mathrm{Z}-\frac{17}{81}\right) & \frac{16}{729} \mathrm{Z} \\
\sqrt{2}\left(\frac{4096}{64827} \mathrm{Z}\right) & \frac{16}{729} \mathrm{Z} & -\mathrm{Z}\left(\frac{5}{8} \mathrm{Z}-\frac{17}{81}\right)
\end{array}\right]
$$


Finally, to calculate the ground state energies of He-like ions, the secular equation in Eq. (13) was then solved as shown in Eq. (25)

$$
\operatorname{det}\left[\begin{array}{ccc}
-Z\left(Z-\frac{5}{8}\right)-E & \sqrt{2}\left(\frac{4096}{64827} Z\right) & \sqrt{2}\left(\frac{4096}{64827} Z\right) \\
\sqrt{2}\left(\frac{4096}{64827} Z\right) & -Z\left(\frac{5}{8} Z-\frac{17}{81}\right)-E & \frac{16}{729} Z \\
\sqrt{2}\left(\frac{4096}{64827} Z\right) & \frac{16}{729} Z & -Z\left(\frac{5}{8} Z-\frac{17}{81}\right)-E
\end{array}\right]=0
$$

Eq. (25) is a very simple expression for energies of helium-like ions because it only depends on the atomic number $\mathrm{Z}$ of the ions. In that equation, an otherwise complicated calculation to solve the Schrödinger equation has been reduced into a simple calculation of the determinant of a $3 \times 3$ matrix. The calculation of the ground state energies of any He-like ion was done by substituting the atomic number $\mathrm{Z}$ of the ions into Eq. (25). The equation was then solved analytically to obtain three energy eigenvalues of the ions, with the lowest energy was the ground state energy. The obtained ground state energies for helium-like ions $(3 \leq Z \leq 10)$ are shown in Table 1.

\section{Numerical Calculation of the Ground State Energies of Helium-like Ions}

As the number of basis state increases, energies are expected to be more accurate because more physical information is included in the wavefunction. It was proven to be valid in the study of the helium atom [21]. It would also make the analytical calculation very inefficient to perform for more than three basis states. Therefore, a numerical calculation using a simple Mathematica code was used here to perform the calculation.

It is important to state the meaning of analytical and numerical terms used in this article. Analytical calculation refers to the calculation by hand involving only 3 basis states and $3 \times 3$ Hamiltonian matrix. Meanwhile, numerical calculation refers to a calculation involving more than 3 basis states, i.e., Hamiltonian of size greater than $3 \times 3$. In addition, the numerical calculation was performed using a Mathematica code involving a matrix of size $25 \times 25$. Here, 25 basis states were used, which can be written as
$|\Psi\rangle=\sum_{\mathrm{n}=2}^{13} \mathrm{c}_{11}|1 \mathrm{~s} 1 \mathrm{~s}\rangle+\mathrm{c}_{1 \mathrm{n}}|1 \mathrm{sns}\rangle+\mathrm{c}_{\mathrm{n} 1}|\mathrm{~ns} 1 \mathrm{~s}\rangle$

and therefore, the Hamiltonian was a $25 \times 25$ matrix, as shown below

$\mathrm{H}=\left[\begin{array}{cccc}\mathrm{H}_{1,1} & \mathrm{H}_{1,2} & \ldots & \mathrm{H}_{1,25} \\ \mathrm{H}_{2,1} & \mathrm{H}_{2,2} & \ldots & \mathrm{H}_{2,25} \\ \ldots & \ldots & \ldots & \ldots \\ \mathrm{H}_{25,1} & \mathrm{H}_{25,2} & \ldots & \mathrm{H}_{25,25}\end{array}\right]$

and the corresponding secular equation was

$\operatorname{det}\left[\begin{array}{cccc}\mathrm{H}_{1,1}-\mathrm{E} & \mathrm{H}_{1,2} & \ldots & \mathrm{H}_{1,25} \\ \mathrm{H}_{2,1} & \mathrm{H}_{2,2}-\mathrm{E} & \ldots & \mathrm{H}_{2,25} \\ \ldots & \ldots & \ldots & \ldots \\ \mathrm{H}_{25,1} & \mathrm{H}_{25,2} & \ldots & \mathrm{H}_{25,25}-\mathrm{E}\end{array}\right]=0$

The calculations of all matrix elements in Eq. (27) using Eq. (11) and the solutions to the secular equation in Eq. (28) were performed using a Mathematica code modified from [20].

All the numerical energies are presented in Table 1 along with energies obtained from other simple approaches available in the literature, such as the geometrical model (GM) [18], the hydrogenic $1^{\text {st }}$ order perturbation theory (SPT) [19], and the uncertainty principle-variational approach (UPV) [23]. The exact ground state energies of the ions were taken from [13] and were used to calculate the percentage errors, as shown in Table 1. Corresponding experimental values for the respective atoms were adapted from [24] and also shown in Table 1.

\section{Comparisons with Ground State Energies using other Simple Methods}

From Table 1, it is clear that our method yielded reasonably accurate ground state energies of helium-like ions. The least accurate result of our method was obtained for the He atom [19], with percentage errors of $2.51 \%$ and $2.02 \%$, respectively, from our analytical and numerical calculations. Although this result for the $\mathrm{He}$ atom was less accurate compared to the geometrical model [18] (0.32\% error), it was still more accurate than the results of the first-order perturbation theory [19] $(5.28 \%$ error) and uncertainty principle-variational approach [23] (2.60\% error). The most accurate results in this article were obtained for the ground state energy 
Table 1. Ground state energies of helium atom and light he-like ions from our analytical calculation (AC), our numerical calculation (NC), the geometrical model (GM) [18], standard first-order perturbation theory (SPT) [19] and the uncertainty principle-variational approach (UPV) [23]. Energies are in atomic units

\begin{tabular}{cccccccc}
\hline $\begin{array}{c}\text { Atom/ } \\
\text { Ion }\end{array}$ & $\begin{array}{c}\mathrm{AC} \\
\text { (\% error) }\end{array}$ & $\begin{array}{c}\mathrm{NC} \\
\text { (\% error) }\end{array}$ & $\begin{array}{c}\mathrm{SPT} \\
\text { (\% error) }\end{array}$ & $\begin{array}{c}\mathrm{GM} \\
\text { (\% error) }\end{array}$ & $\begin{array}{c}\mathrm{UPV} \\
(\% \text { error) }\end{array}$ & $\begin{array}{c}\text { Exact } \\
{[13]}\end{array}$ & $\begin{array}{c}\text { Experiment } \\
{[24]}\end{array}$ \\
\hline \multirow{2}{*}{$\mathrm{He}$} & $-2.8304^{\ddagger}$ & $-2.8446^{+}$ & -2.7500 & -2.9128 & -2.8279 & -2.9034 & -2.90338 \\
& $(2.51 \%)$ & $(2.02 \%)$ & $(5.28 \%)$ & $\begin{array}{c}(0.32 \%) \\
(2.60 \%)\end{array}$ & & \\
& -7.1886 & -7.2053 & -7.1250 & -7.3249 & -7.1880 & -7.2799 & -7.2790 \\
$\mathrm{Li}^{+}$ & $(1.25 \%)$ & $(1.02 \%)$ & $(2.16 \%)$ & $(0.62 \%)$ & $(1.26 \%)$ & & \\
& -13.5570 & -13.5740 & -13.5000 & -13.7373 & -13.5486 & -13.6556 & -13.6574 \\
$\mathrm{Be}^{2+}$ & $(0.72 \%)$ & $(0.60 \%)$ & $(1.18 \%)$ & $(0.60 \%)$ & $(0.78 \%)$ & & \\
& -21.9285 & -21.9455 & -21.8750 & -22.1477 & -21.9083 & -22.0310 & -22.0360 \\
$\mathrm{~B}^{3+}$ & $(0.47 \%)$ & $(0.39 \%)$ & $(0.75 \%)$ & $(0.53 \%)$ & $(0.56 \%)$ & & \\
& -32.3014 & -32.3183 & -32.2500 & -32.5573 & -32.2672 & -32.4062 & -32.4174 \\
$\mathrm{C}^{4+}$ & $(0.32 \%)$ & $(0.27 \%)$ & $(0.52 \%)$ & $(0.47 \%)$ & $(0.43 \%)$ & & \\
& -44.6749 & -44.6918 & -44.6250 & -44.9661 & -44.6252 & -44.7814 & -44.8035 \\
$\mathrm{~N}^{5+}$ & $(0.24 \%)$ & $(0.20 \%)$ & $(0.39 \%)$ & $(0.41 \%)$ & $(0.35 \%)$ & & \\
& -59.0489 & -59.0657 & -59.0000 & -59.3751 & -58.9824 & -59.1566 & -59.1958 \\
$\mathrm{O}^{6+}$ & $(0.18 \%)$ & $(0.15 \%)$ & $(0.31 \%)$ & $(0.37 \%)$ & $(0.29 \%)$ & & \\
& -75.4231 & -75.4398 & -75.3750 & -75.7833 & -75.3388 & -75.5317 & -75.5441 \\
$\mathrm{~F}^{7+}$ & $(0.14 \%)$ & $(0.12 \%)$ & $(0.25 \%)$ & $(0.33 \%)$ & $(0.26 \%)$ & & \\
& -93.7975 & -93.8142 & -93.7500 & - & -93.6943 & -93.9068 & -94.0086 \\
$\mathrm{Ne}^{8+}$ & $(0.12 \%)$ & $(0.10 \%)$ & $(0.21 \%)$ & $(-)$ & $(0.23 \%)$ & & \\
\hline
\end{tabular}

tenergies were taken from our previous calculation in [21]

of $\mathrm{Ne}^{8+}$, with errors reaching as small as $0.12 \%$ from our analytical calculations and $0.10 \%$ from our numerical calculations, which are more accurate than standard perturbation theory, uncertainty principle-variational approach, and the geometrical model. The comparisons of the percentage errors of our analytical (AC) and numerical energies (NC) with other relatively simple calculations are shown in Fig. 1.

It should be noted that although our model generated more accurate energies than the geometrical model for $\mathrm{Z}>4$, the geometrical model was much more accurate than ours for $Z=2$ and $Z=3$. In particular, $\%$ errors from our numeric calculation were about 6.3 times larger than that from the geometrical model for the helium atom and approximately 1.6 times larger for the $\mathrm{Li}^{+}$ion. This strongly indicates that a general conclusion about whether our model or the geometrical model was more accurate in calculating ground state energies of twoelectron systems can not be drawn. On the other hand, our model was more accurate than the standard perturbation theory calculated in [19] and the uncertainty principle variational calculation presented in [23].

Fig. 1 clearly shows the numerical results are more accurate than the analytical ones, especially for small $\mathrm{Z}$. The accuracy is the result of the 25 basis states used in the numeric calculation, compared to only three basis states in the analytic one. For larger atomic number, the differences between the two become less obvious. It indicates that adding the number of basis states does not significantly impact the accuracy of our calculations for ions with a relatively large atomic number. The discussion on this is presented in the following section.

As shown in Fig. 1, the results indicate that errors from our analytical and numerical calculations for all ions were much smaller than those from standard perturbation theory [19] and the uncertainty principlevariational approach [23]. In particular, for ions with a small atomic number such as $\mathrm{Li}^{+}$and $\mathrm{Be}^{2+}$, calculation using the standard perturbation theory gives errors of 


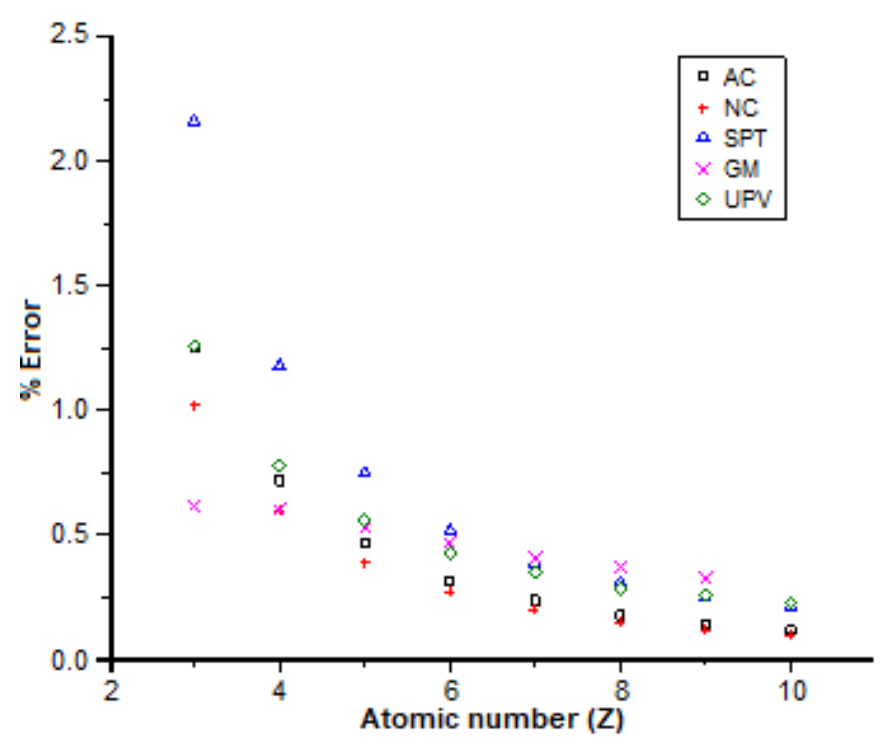

Fig 1. Percentage errors of our calculation (AC and NC) are compared with those from standard perturbation theory (SPT) [19], the geometrical model (GM) [18], and the uncertainty principle-variational approach (UPV) [23] as a function of atomic number $\mathrm{Z}(3 \leq \mathrm{Z} \leq 10)$

about $2.16 \%$ and $1.18 \%$, respectively. Errors from our method for the respective ions are $1.25 \%$ and $0.72 \%$ (analytic) and $1.02 \%$ and $0.60 \%$ (numeric). These results indicate that our method significantly reduces errors obtained from standard perturbation theory for He-like ions, especially for ions with relatively small atomic number Z. Errors from our numeric energies for all ions are also much lower than errors found in [23]. Although our analytic calculation for $\mathrm{Li}^{+}$ion has approximately the same error as in [23], as atomic number increases, error from our calculations becomes much smaller than that from their calculations. Furthermore, comparisons to the geometrical model [18] show that for $\mathrm{Li}^{+}(\mathrm{Z}=3)$, our energy calculation is less accurate. For $\mathrm{Be}^{2+}(Z=4)$, the error from our numerical calculation is the same as that from the geometrical model. For $\mathrm{Z}>4\left(\mathrm{~B}^{3+}\right.$ through $\left.\mathrm{F}^{7+}\right)$, all our analytical and numerical energies are much better than those from the geometrical model. It also indicates that as nuclear charge increases, our approach becomes much more powerful than the geometrical model.

It should be noted that the accuracy of our results (which did not use any variational parameter) is, of course, not to be compared with the accuracy of calculations using several or even hundreds to thousands of variational parameters such as the work of [3,25-29] which reproduced experimental values and exact values of Pekeris [13]. As stated in the introduction, our simple calculation in this paper was only intended to demonstrate that using the hydrogenic orbitals without correlation function in a simple matrix calculation could be more accurate than some other simple methods in the literature.

\section{Percentage Errors as a Function of Atomic Number Z}

Table 1 also shows that the percentage errors decrease as the atomic number of the ions increases. The results were reliable because as the atomic number increases, electrostatic interaction between nuclei and electrons becomes more dominant compared to mutual interaction between the two electrons [19]. Most recently, Rahman et al. [30] calculated the electronelectron repulsion energies and Coulomb ionic energies of the $\mathrm{He}$ atom and some He-like ions $\left(\mathrm{Li}^{+}\right.$and $\left.\mathrm{Be}^{2+}\right)$ and found that as atomic number increases, the increase in the ionic energies of the ions was much more significant than that in the electron-electron repulsion energies. They found that the ionic energies of $\mathrm{He}, \mathrm{Li}^{+}$and $\mathrm{Be}^{2+}$ were -6.7533 a.u., -16.1275 a.u., and -29.5020 a.u., respectively, while the repulsion energies of the respective ions were 0.9458 a.u., 1.5677 a.u., and 2.1909 a.u. It is clear from these results that the increase in the repulsion energies was much less than the increase in the ionic energies as $\mathrm{Z}$ increases. This was caused by the fact that electrons are more localized near higher $\mathrm{Z}$ ions [30]. Therefore, the fact that our approximation does not include electron correlation function in the wavefunction has led to lower percentage errors in ground state energies for ions with larger atomic numbers has already been anticipated.

To further clarify this, the probability amplitudes for each state used in the wave function expansion in our analytic and numerical calculation were calculated and presented in Table 2.

There is a very clear indication from Table 2 that the largest contribution to the ground state energy of the 
Table 2. Amplitudes $c_{1 i}=c_{i 1}$ of the states used in the wavefunction expansion $\left|\mathrm{n}_{1}, \mathrm{l}_{1}, \mathrm{~m}_{11} ; \mathrm{n}_{2}, \mathrm{l}_{2}, \mathrm{~m}_{12}\right\rangle$ of our analytical and numerical calculations

\begin{tabular}{|c|c|c|c|c|c|c|c|c|}
\hline \multicolumn{9}{|c|}{ Analytical Calculation } \\
\hline State & $\mathrm{Li}^{+}$ & $\mathrm{Be}^{2+}$ & $\mathrm{B}^{3+}$ & $\mathrm{C}^{4+}$ & $\mathrm{N}^{5+}$ & $\mathrm{O}^{6+}$ & $\mathrm{F}^{7+}$ & $\mathrm{Ne}^{8+}$ \\
\hline$|1,0,0 ; 1,0,0\rangle$ & -0.9862 & -0.9937 & -0.9964 & -0.9977 & -0.9984 & -0.9988 & -0.9991 & -0.9993 \\
\hline$|1,0,0 ; 2,0,0\rangle$ & 0.1170 & 0.0792 & 0.0597 & 0.0478 & 0.0398 & 0.0342 & 0.0299 & 0.0266 \\
\hline$|2,0,0 ; 1,0,0\rangle$ & 0.1170 & 0.0792 & 0.0597 & 0.0478 & 0.0398 & 0.0342 & 0.0299 & 0.0266 \\
\hline \multicolumn{9}{|c|}{ Numerical Calculation } \\
\hline State & $\mathrm{Li}^{+}$ & $\mathrm{Be}^{2+}$ & $\mathrm{B}^{3+}$ & $\mathrm{C}^{4+}$ & $\mathrm{N}^{5+}$ & $\mathrm{O}^{6+}$ & $\mathrm{F}^{7+}$ & $\mathrm{Ne}^{8+}$ \\
\hline$|1,0,0 ; 1,0,0\rangle$ & 0.9847 & 0.9927 & 0.9957 & 0.9972 & 0.9980 & 0.9985 & 0.9989 & 0.9991 \\
\hline$|1,0,0 ; 2,0,0\rangle$ & -0.1101 & -0.0760 & -0.0578 & -0.0466 & -0.0390 & -0.0336 & -0.0294 & -0.0262 \\
\hline$|2,0,0 ; 1,0,0\rangle$ & -0.1101 & -0.0760 & -0.0578 & -0.0466 & -0.0390 & -0.0336 & -0.0294 & -0.0262 \\
\hline$|1,0,0 ; 3,0,0\rangle$ & -0.0415 & -0.0296 & -0.0229 & -0.0186 & -0.0157 & -0.0136 & -0.0119 & -0.0107 \\
\hline$|3,0,0 ; 1,0,0\rangle$ & -0.0415 & -0.0296 & -0.0229 & -0.0186 & -0.0157 & -0.0136 & -0.0119 & -0.0107 \\
\hline$|1,0,0 ; 4,0,0\rangle$ & -0.0243 & -0.0174 & -0.0135 & -0.0110 & -0.0093 & -0.0080 & -0.0071 & -0.0063 \\
\hline$|4,0,0 ; 1,0,0\rangle$ & -0.0243 & -0.0174 & -0.0135 & -0.0110 & -0.0093 & -0.0080 & -0.0071 & -0.0063 \\
\hline$|1,0,0 ; 5,0,0\rangle$ & -0.0167 & -0.0120 & -0.0093 & -0.0076 & -0.0064 & -0.0055 & -0.0049 & -0.0043 \\
\hline$|5,0,0 ; 1,0,0\rangle$ & -0.0167 & -0.0120 & -0.0093 & -0.0076 & -0.0064 & -0.0055 & -0.0049 & -0.0043 \\
\hline$|1,0,0 ; 6,0,0\rangle$ & -0.0124 & -0.0089 & -0.0069 & -0.0056 & -0.0048 & -0.0041 & -0.0036 & -0.0032 \\
\hline$|6,0,0 ; 1,0,0\rangle$ & -0.0124 & -0.0089 & -0.0069 & -0.0056 & -0.0048 & -0.0041 & -0.0036 & -0.0032 \\
\hline$|1,0,0 ; 7,0,0\rangle$ & -0.0098 & -0.0070 & -0.0054 & -0.0044 & -0.0037 & -0.0032 & -0.0028 & -0.0025 \\
\hline$|7,0,0 ; 1,0,0\rangle$ & -0.0098 & -0.0070 & -0.0054 & -0.0044 & -0.0037 & -0.0032 & -0.0028 & -0.0025 \\
\hline$|1,0,0 ; 8,0,0\rangle$ & -0.0079 & -0.0057 & -0.0044 & -0.0036 & -0.0030 & -0.0026 & -0.0023 & -0.0021 \\
\hline$|8,0,0 ; 1,0,0\rangle$ & -0.0079 & -0.0057 & -0.0044 & -0.0036 & -0.0030 & -0.0026 & -0.0023 & -0.0021 \\
\hline$|1,0,0 ; 9,0,0\rangle$ & -0.0066 & -0.0047 & -0.0037 & -0.0030 & -0.0025 & -0.0022 & -0.0019 & -0.0017 \\
\hline$|9,0,0 ; 1,0,0\rangle$ & -0.0066 & -0.0047 & -0.0037 & -0.0030 & -0.0025 & -0.0022 & -0.0019 & -0.0017 \\
\hline$|1,0,0 ; 10,0,0\rangle$ & -0.0057 & -0.0040 & -0.0031 & -0.0026 & -0.0022 & -0.0019 & -0.0016 & -0.0015 \\
\hline$|10,0,0 ; 1,0,0\rangle$ & -0.0057 & -0.0040 & -0.0031 & -0.0026 & -0.0022 & -0.0019 & -0.0016 & -0.0015 \\
\hline$|1,0,0 ; 11,0,0\rangle$ & -0.0049 & -0.0035 & -0.0027 & -0.0022 & -0.0019 & -0.0016 & -0.0014 & -0.0013 \\
\hline$|11,0,0 ; 1,0,0\rangle$ & -0.0049 & -0.0035 & -0.0027 & -0.0022 & -0.0019 & -0.0016 & -0.0014 & -0.0013 \\
\hline$|1,0,0 ; 12,0,0\rangle$ & -0.0043 & -0.0031 & -0.0024 & -0.0019 & -0.0016 & -0.0014 & -0.0012 & -0.0011 \\
\hline$|12,0,0 ; 1,0,0\rangle$ & -0.0043 & -0.0031 & -0.0024 & -0.0019 & -0.0016 & -0.0014 & -0.0012 & -0.0011 \\
\hline$|1,0,0 ; 13,0,0\rangle$ & -0.0038 & -0.0027 & -0.0021 & -0.0017 & -0.0014 & -0.0012 & -0.0011 & -0.0010 \\
\hline$|13,0,0 ; 1,0,0\rangle$ & -0.0038 & -0.0027 & -0.0021 & -0.0017 & -0.0014 & -0.0012 & -0.0011 & -0.0010 \\
\hline
\end{tabular}

ions came from the $|1,0,0 ; 1,0,0\rangle$ state, i.e. when the two electrons are in the ground state of the ions. This can be seen from the probability amplitude values $\left(\mathrm{c}_{11}^{2}\right)$ for the $|1,0,0 ; 1,0,0\rangle$ state which reached about $97.26 \%$ and $96.96 \%$ from our analytic and numeric calculations, respectively, for $\mathrm{Li}^{+}$ion. A similar calculation was performed by [31], who found that the largest contribution to the ground state energy of the He atom was from $|1,0,0 ; 1,0,0\rangle$ state (about 93\%). Hutchinson et al. [31] found that the remaining contribution to the ground state energy of the
He atom came from the higher 1 bound states as well as the unbound states of the atom. This is also in agreement with our results, as shown in Table 2. The importance of including hydrogenic unbound states and the bound states in the wavefunction was also discussed in [32-33], which also showed that reasonably accurate ground state energies of two-electron atoms could be generated by the matrix mechanics utilizing hydrogenic matrix mechanics. These studies supported the reasonably accurate results obtained in this article for he-like ions. 
Meanwhile, Table 2 also indicated that the contribution from higher states was much smaller compared to the $|1,0,0 ; 1,0,0\rangle$ with the lowest one came from the $|1,0,0 ; 2,0,0\rangle$ state $\left(c_{12}^{2}=1.37 \%\right)$ for the analytic calculation and $c_{1,13}^{2}=1.44 \times 10^{-3} \%$ for the $|1,0,0 ; 13,0,0\rangle$ state from the numeric calculation for $\mathrm{Li}^{+}$ion. Furthermore, it is clear from Table 2 that as the atomic number $Z$ of the ions increases, the contribution of $\mid 1,0,0$; $1,0,0\rangle$ state to the ground state energies of the ions also increases while at the same time the contribution of the higher states decreases. For instance, the contribution was about $96.96 \%$ for $\mathrm{Li}^{+}$ion $(\mathrm{Z}=3)$, which increased and reached about $99.82 \%$ for $\mathrm{Ne}^{8+}$ ion $(Z=10)$. A similar finding was also reported by Tapilin [24,34], who used configuration weight function and showed that in all cases considered, the $|1,0,0 ; 1,0,0\rangle$ configuration was an increasing function. He also showed that the growth of the $|1,0,0 ; 1,0,0\rangle$ configuration slowed down with increasing nuclear charge $\mathrm{Z}$ and tended to be constant. This is in agreement with our results shown in Table 2 and Fig. 2. This also explains why energies from our calculation for larger $\mathrm{Z}$ ions were more accurate than those for lower $\mathrm{Z}$ ions, as shown in Table 1 and Fig. 1. This finding is visualized in Fig. 2, where probability amplitudes of the states used in the wave function expansion were plotted as a function of atomic number $\mathrm{Z}$ for analytic calculation (Fig. 2(a)) and numeric calculation (Fig. 2(b)).

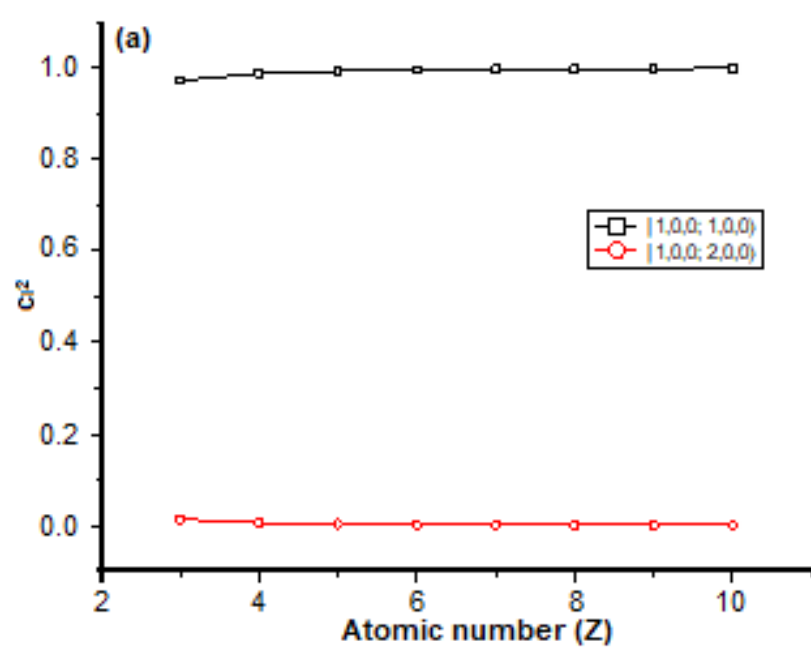

Fig. 2 demonstrates that contribution from the $|1,0,0 ; 1,0,0\rangle$ state when using 3 basis states in our analytical calculation was approximately the same as that when using 25 basis states for a particular ion. There was a slight increase in the probability amplitudes of the state, but since the probability amplitudes almost reached $100 \%$, further addition of hydrogenic s-bound states in the wave function using approximation here would not significantly improve the accuracy of our calculation. The use of correlation function involving variational parameters should therefore be included to significantly improve the results of our calculations, which has been performed using various method and widely reported in the literature. Including the correlation function and variational parameters would improve the accuracy. However, at the same time, the difficulty levels of the calculations increase, which was out of the scope of the present research. Calculation of electron correlation in $\mathrm{He}$ and He-like ions can be found, for example, in [35], which used the Laguerrebased wave function to obtain the fully correlated wave function and the Hartree-Fock wave function. A similar calculation on the He atom using a modified Hylleraas trial function containing two linear parameters was also reported recently by Purwaningsih et al. [36].

Table 2 also clearly indicates that as the nuclear charge $\mathrm{Z}$ increased, the contribution from higher $\mathrm{n}$ states

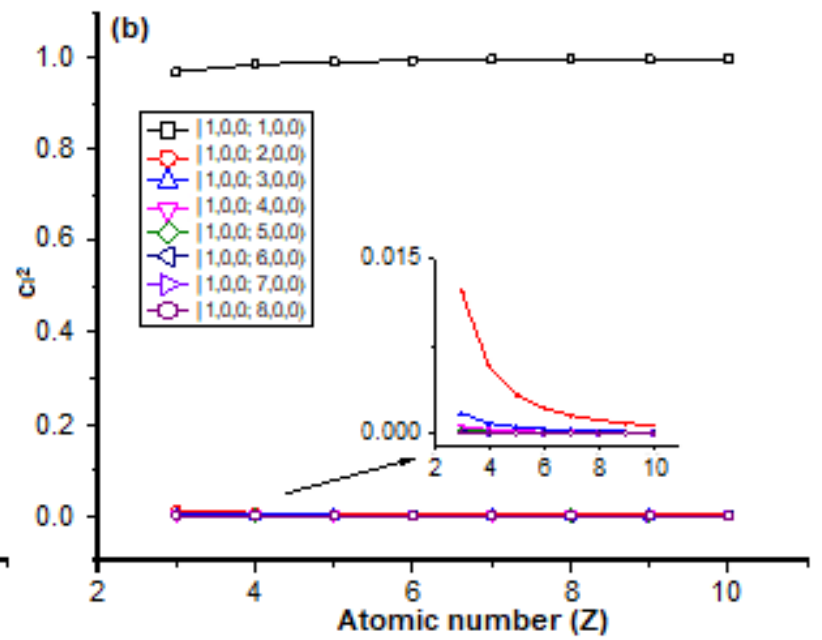

Fig 2. Probability amplitudes $c_{1 i}^{2}$ of states used in the wave function was plotted against the ion atomic number $\mathrm{Z}$ for the analytic calculation (a) and numeric calculation (b). Since $|1,0,0 ; n, 0,0\rangle$ and $|n, 0,0 ; 1,0,0\rangle$ states were equivalent, only $|1,0,0 ; n, 0,0\rangle$ states were shown 
to the ground state energies of the ions decreased. This was expected because the same number of basis states were used in our calculation, meaning that the larger the contribution from the ground state configuration $\mid 1,0,0$; $1,0,0\rangle$ for a particular ion should lead to the smaller contribution from the higher $\mathrm{n}$-state configuration $\mid 1,0,0$; $\mathrm{n}, 0,0\rangle$. The probability amplitudes continued to decrease for larger $\mathrm{n}$ for a particular ion for a particular matrix size.

\section{Comments on the Accuracy and Limitations of Our Method and Further Improvement}

The fact that our matrix method based on hydrogenic orbitals could generate reasonably accurate results with errors not larger than 1.02\% for helium-like ions with $Z \geq 3$ was expected since many studies have shown that the use of (modified) hydrogenic wave functions could produce accurate results. For instance, studies by [20] and our previous work [21-22] showed that the reasonably accurate ground state energy of the $\mathrm{He}$ atom and the first excited state energy of some light Helike ions could be obtained using this method. In addition, the use of $|1,0,0 ; n, 0,0\rangle$ states, i.e. one electron was kept at the ground states, and the other one in a particular excited state $\mathrm{n}$ was used by Hall and Siegel [37], who applied the shooting method to obtain accurate excited state energies of the $\mathrm{He}$ atom in coordinate space. This type of state configuration was also used as a pseudo-two-body problem by Gueribah et al. [38], who used the experimental energies to determine the local equivalent potential of the He atom using the Heisenberg model. Moreover, was applied by Herschbach et al. [39] applied the scaling dimension technique to obtain accurate ground state energies of two-electron atoms based on the dimensional dependence of a hydrogen atom. In their study, zeroth-order hydrogenic wave function was used to evaluate the expectation values of the electron-electron repulsion in the two-electron atoms. Finally, Rahman et al. [40] used the linear combination of hydrogenic wave functions to obtain accurate energy levels of hydrogenic ions by means of an iteration technique. Their method was also extended to many-body problems and proven to be accurate for the calculation of the helium-like ions, especially the hydrogen anion.

Since the results from our simple matrix calculation were reasonably accurate, the matrix method can be extended to study the chemical and physical properties of other systems. This was already done in some recent studies, such as in [41-43]. However, since we only used s-states $|1,0,0 ; \mathrm{n}, 0,0\rangle$ in our wave function, results in this article could be improved by including higher bound lstates $\left|1,0,0 ; \mathrm{n}, 1, \mathrm{~m}_{1}\right\rangle$ and continuum states. This would be our next focus on this topic. In addition, since no variational parameter and correlation function were involved in our calculation, results from our calculation were expected to have larger errors than those from other calculations involving variational parameters and correlation function. However, we have successfully shown that for ions with $Z>4$, our parameter-free matrix results were more accurate than the geometrical model [18], which used one variational parameter in the model to calculate the ground state energy of the ions.

\section{- CONCLUSION}

A parameter-free matrix method based on hydrogenic orbital approximations has been successfully applied in the present study to accurately calculate the ground state energies of helium-like ions from $\mathrm{Li}^{+}$to $\mathrm{Ne}^{8+}$. A new and very simple analytic expression for the ground state energy of any helium-like ion was derived and has been proven to be reasonably accurate. A simple numerical calculation was also applied to improve the accuracy of analytical energies. Despite being simple, our results for all ions are much more accurate than some other simple methods available in the literature, such as the standard hydrogenic perturbation theory and the uncertainty principle-variational approach. In addition, our results are also more accurate than the geometrical model for $\mathrm{Z}>4\left(\mathrm{~B}^{3+}\right.$ through $\left.\mathrm{F}^{7+}\right)$. Therefore, our method can be introduced as an alternative and reasonably accurate method to obtain ground state energies of two-electron atoms with less algebra and inexpensive computer calculations.

\section{- ACKNOWLEDGMENTS}

The authors thank Prof. Thad G. Walker and Dr. Robert Masse for the permission to modify their Mathematica code to perform the numerical calculation in this article. We also thank Prof. Christopher T. 
Chantler for his comments on our manuscript. This research was a part of research funded by PNBP Researchgrant 2021 No. 41/UN15.15.2.PPK/SPP/FST/IV/2021 of The Faculty of Science and Engineering, University of Nusa Cendana.

\section{- AUTHOR CONTRIBUTIONS}

RKP wrote the original draft of the manuscript and performed the calculation. RKP, AA, and UAD analyzed and interpreted the results as well as revised the manuscript. All authors agreed to the final version of this manuscript.

\section{- REFERENCES}

[1] Paterson, D., Chantler, C.T., Hudson, L.T., Serpa, F.F., Gillaspy, J.D., and Takacs, E., 2001, Absolute Test of Quantum Electrodynamics for Helium-Like Vanadium" in The Hydrogen Atom, Lecture Notes in Physics Vol 570, Eds. Karshenboim, S.G., Bassani, F., Pavone, F., Inguscio, M., Hansch, T., (eds.), Springer, Berlin, Heidelberg, 699-713.

[2] Yerokhin, V.A., and Pachucki, K., 2010, Theoretical energies of low-lying states of light helium-like ions, Phys. Rev. A, 81 (2), 022507.

[3] Tupitsyn, I.I., Bezborodov, S.V., Malyshev, A.V., Mironova, D.V., and Shabaev, V.M., 2020, Calculations of relativistic, correlation, nuclear, and quantum-electrodynamics corrections to energy and ionization potential of the ground state of heliumlike ions, Opt. Spectrosc., 128 (1), 21-31.

[4] Cioslowski, J., and Prątnicki, F., 2020, Uniform description of the helium isoelectronic series down to the critical nuclear charge with explicitly correlated basis sets derived from regularized Krylov sequences, J. Chem. Phys., 153 (22), 224106.

[5] Cioslowski, J., and Prątnicki, F., 2019, Natural amplitudes of the ground state of the helium atom: Benchmark calculations and their relevance to the issue of unoccupied natural orbitals in the $\mathrm{H}_{2}$ molecule, J. Chem. Phys., 150 (7), 074111.

[6] Cioslowski, J., and Prątnicki, F., 2018, Simpler is often better: Computational efficiency of explicitly correlated two-electron basis sets generated by the regularized Krylov sequences of Nakatsuji, J. Chem. Phys., 149 (18), 184107.

[7] Stoyanov, Z.K., Pavlov, R., Mihailov, L.M., Velchev, Ch.J., Mutafchieva, Y.D., Tonev, D., and Chamel, N., 2016, Nuclear induces effects and mass correlations in low and multiply charged heliumlike ions, J. Phys. Conf. Ser., 724, 012048.

[8] Velchev, C.J., Pavlov, R., Tonev, D., Stoyanov, Z.K., Mihailov, L.M., Mutafchieva, Y.D., and Van Neck, D., 2017, "Effects of Isotope Characteristics on the Electron System Ground State Energy of HeliumLike Ions" in Quantum Systems in Physics, Chemistry, and Biology. Progress in Theoretical Chemistry and Physics Vol 30, Eds. Tadjer, A., Pavlov, R., Maruani, J., Brändas, E., DelgadoBarrio, G., Springer, Cham, 283-300.

[9] Nakashima, H., and Nakatsuji, H., 2007, Solving the Schrödinger equation for helium atom and its isoelectronic ions with the free iterative complement interaction (ICI) method, J. Chem. Phys., 127 (22), 224104.

[10] Nakashima, H., and Nakatsuji, H., 2008, Solving the electron-nuclear Schrödinger equation of helium atom and its isoelectronic ions with the freeiterative-complement interaction method, J. Chem. Phys., 128 (15), 154107.

[11] Turbiner, A.V., Vieyra, J.C.L., and Olivares-Pilòn, H., 2019, Few-electron atomic ions in non-relativistic QED: The ground state, Ann. Phys., 409, 167908.

[12] Turbiner, A.V., Vieyra, J.C.L., del Valle, J.C., and Nader, D.J., 2020, Ultra-compact accurate wave functions for He-like and Li-like iso-electronic sequences and variational calculus: I. Ground state, Int. J. Quantum Chem., 121 (8), e26586.

[13] Pekeris, C.L., 1958, Ground states of two-electron atoms, Phys. Rev., 112 (5), 1649-1658.

[14] Rodriguez, K.V., Gasaneo, G., and Mitnik, D.M., 2007, Accurate and simple wavefunctions for the helium isoelectronic sequence with correct cusp conditions, J. Phys. B: At., Mol. Opt. Phys., 40 (19), 3923-3939. 
[15] Liverts, E.Z., and Barnea, N., 2011, S-States of helium-like ions, Comput. Phys. Commun., 182 (9), 1790-1795.

[16] Ancarani, L.U., Rodriguez, K.V., and Gasaneo, G., 2007, A simple parameter-free wavefunction for the ground state of two-electron atoms, J. Phys. B: At. Mol. Opt. Phys., 40 (13), 2695-2702.

[17] Utomo, Y.R., Maruto, G., Utomo, A.B.S., Nurwantoro, P., and Sholihun, S., 2020, Numerical calculation of energy eigen-values of the hydrogen negative ion in the $2 \mathrm{p}^{2}$ configuration by using the variational method, J. Fis. Indones., 24 (1), 30-32.

[18] Gomez, R.W., 1992, Ground and excited energy levels of helium-like atoms using a simple geometrical model, Eur. J. Phys., 13 (3), 135-138.

[19] Bransden, B.H., and Joachain, C.J., 2000, Quantum Mechanics, $2^{\text {nd }} E d$., Prentice Hall, Hoboken, US.

[20] Massé, R., and Walker, T.G., 2015, Accurate energies of the $\mathrm{He}$ atom with undergraduate quantum mechanics, Am. J. Phys., 83 (8), 730-732.

[21] Pingak, R.K., Kolmate, R., and Bernandus., 2019, A simple matrix approach to determination of the helium atom energies, JPFA, 9 (1), 10-21.

[22] Pingak, R.K., and Deta, U.A., 2020, A simple numerical matrix method for accurate triplet- $1 \mathrm{~s} 2 \mathrm{~s}{ }^{3} \mathrm{~S}_{1}$ energy levels of some light helium-like ions, J. Phys. Conf. Ser., 1491, 012035.

[23] Harbola, V., 2011, Using uncertainty principle to find the ground-state energy of the helium and a helium-like Hookean atom, Eur. J. Phys., 32 (6), 1607-1615.

[24] Tapilin, V.M., 2019, Solving the Schrodinger equation for helium-like ions with the method of configuration weight functions, J. Struct. Chem., 60 (1), 1-6.

[25] King, A.W., Baskerville, A.L., and Cox, H., 2018, Hartree-Fock implementation using a Laguerrebased wave function for the ground state and correlation energies of two-electron atoms, Philos. Trans. R. Soc., A, 376 (2115), 20170153.

[26] Aznabaev, D.T., Bekbaev, A.K., Ishmukhamedov, I.S., and Korobov, V.I., 2015, Energy levels of a helium atom, Phys. Part. Nucl. Lett., 12 (5), 689-694.
[27] Chen, Y.H., and Chao, S.D., 2017, Kinetic energy partition method applied to ground state heliumlike atoms, J. Chem. Phys., 146 (12), 124120.

[28] Aznabaev, D.T., Bekbaev, A.K., and Korobov, V.I., 2018, Nonrelativistic energy levels of helium atoms, Phys. Rev. A, 98, 012510.

[29] Yerokhin, V.A., Patkóš, V., Puchalski, M., and Pachucki, K., 2020, QED calculation of ionization energies of 1 snd states in helium, Phys. Rev. A, 102, 012807.

[30] Rahman, F.U., Sarwono, Y.P., and Zhang, R.Q., 2021, Solution of two-electron Schrodinger equations using a residual minimization method and one-dimensional basis functions, AIP $A d v ., 11$ (2), 025228 .

[31] Hutchinson, J., Baker, M., and Marsiglio, F., 2013, The spectral decomposition of the helium atom two-electron configuration in terms of hydrogenic orbitals, Eur. J. Phys., 34, 111-128.

[32] Di Rocco, H.O., 2013, Comment on 'The spectral decomposition of the helium atom two-electron configuration in terms of hydrogenic orbitals', Eur. J. Phys., 34 (2), L43.

[33] Forestell, L., and Marsiglio, F., 2015, The importance of basis states: An example using the hydrogen basis, Can. J. Phys., 93 (10), 1009-1014.

[34] Tapilin, V.M., 2019, A new method of solving the many-body Schrodinger equation, J. Struct. Chem., $58,1-8$.

[35] Baskerville, A.L., King, A.W., and Cox, H., 2019, Electron correlation in $\mathrm{Li}^{+}, \mathrm{He}, \mathrm{H}^{-}$and the critical nuclear charge system $Z_{C}$ : Energies, densities and Coulumb holes, R. Soc. Open Sci., 6 (1), 181357.

[36] Purwaningsih, S., Nurwantoro, P., and Hermanto, A., 2019, Calculation of ground state energy of helium using Hylleraas trial function expansion, Int. J. Eng. Res. Sci. Technol., 12 (8), 1178-1182.

[37] Hall, S., and Siegel, P.B., 2015, Calculating helium atomic excited states in coordinate space, Am. J. Phys., 83 (12), 1028-1038.

[38] Gueribah, S., Ighezou, E.Z., Lombard, R.J., and Ngo, H., 2011, The Heisenberg model of the $\mathrm{He}$ atom revisited, Few-Body Syst., 51, 59-67. 
[39] Herschbach, D.R., Loeser, J.G., and Virgo, W.L., 2017, Exploring unorthodox dimensions for two-electron atoms, J. Phys. Chem. A, 121 (33), 6336-6340.

[40] Rahman, F.U., Zhao, R., Sarwono, Y.P., and Zhang, R.Q., 2018, A scheme of numerical solution for threedimensional isoelectronic series of hydrogen atom using one-dimensional basis functions, Int. J. Quantum Chem., 118 (19), e25694.

[41] Le Vot, F., Meléndez, J.J., and Yuste, S.B., 2016,
Numerical matrix method for quantum periodic potentials, Am. J. Phys., 84 (6), 426-433.

[42] Pavelich, R.L., and Marsiglio, F., 2015, The KronigPenney model extended to arbitrary potentials via numerical matrix mechanics, Am. J. Phys., 83 (9), 773-781.

[43] Jugdutt, B.A., and Marsiglio, F., 2013, Solving for three-dimensional central potentials using numerical matrix methods, Am. J. Phys., 81 (5), 343-350. 